\title{
Uncertainty Evaluation in Determination of Chromium by Inductively Coupled Plasma Mass Spectrometry(ICP-MS)
}

\author{
Zepeng Jiao, Huanyong Li *, Lin Wang, Lvhuan Wang \\ Analytical and Testing Center, Jinan University, Guangzhou 510632, China \\ *Corresponding email: tlihuanyong@jnu.edu.cn
}

Keywords: Chromium, plastic , microwave digestion, ICP-MS, uncertainty

\begin{abstract}
The content of Chromium in plastic products is $(276 \pm 6) \mathrm{mg} / \mathrm{kg}$, which is determinated by Inductively Coupled Plasma Mass Spectrometry (ICP-MS) using Microwave digestion. A mathematical model of the uncertainty containing variables such as regression equation, constant volume, weighing weight and so on is established.
\end{abstract}

\section{Introduction}

Plastic products have deeply affected our lives. Although"the plastic limit order" (which has been implemented for more than a decade) has greatly reduced the white pollution[1-3], it is inevitable that a large number of plastic products still exist and cannot be eliminated. Poor-quality plastic products contain a large amount of heavy metal ions, which greatly affect people's health. They also pollute the environment after they are discarded. Therefore, the detection of heavy metals in such samples has always been the focus of the inspection industry. The evaluation of heavy metal content, which is an important indicator of the manufacturer's product quality. In this paper, a mathematical model of the uncertainty of Chromium is established[4].

\section{Methods and Results}

\subsection{Measurement methods}

$0.2 \mathrm{~g}$ sample was treated by nitric acid with microwave digestion for three times, and decomposed into a $50 \mathrm{~mL}$ volumetric flask.

$0,1,2,3,4,5 \mathrm{~mL}$ standard reserve solution(1 mg/L), which is prepared from $1000 \mathrm{ppm}$ solution, was removed by $1 \mathrm{~mL}$ pipette, respectively, and then set the volume to $100 \mathrm{~mL}$. The intensity of the calibration solution is measured under the same conditions by ICP-MS for three times.

\subsection{Results}

The content of the element Chromium is $278.626,276.685,273.684 \mathrm{mg} / \mathrm{kg}$, respectively.

\section{Mathematical model of uncertainty(Identification and Evaluation of Uncertainty Components)}

Calculation formula of element content is:

$$
\omega=\frac{\mathrm{cV}}{\mathrm{m}}
$$

$\omega(\mathrm{mg} / \mathrm{kg}), \mathrm{c}(\mathrm{mg} / \mathrm{L}), \mathrm{V}(\mathrm{mL}), \mathrm{m}$ :The quality of the sample(g).

\subsection{Source of uncertainty}

The sources of uncertainty by the mathematical model include:

Uncertainty in the concentration of elements in a sample solution based on standard curve line :u(c);

Uncertainty in the concentration of elements in a sample solution based on the sample 
volume:u(V);

Uncertainty of the quality of the sample:u(m);

Uncertainty of repeated experiments:u(rep).

\section{Evaluation of Relative Standard Uncertainty Components}

4.1 Evaluation of relative standard uncertainty of concentration in solution: $u_{\text {rel }}(c)$

4.1.1 Relative standard uncertainty of linear fitting of calibration curve: $u_{\text {rel }}(c)_{1}$

From calibration curve $\mathrm{I}=\mathrm{a}+\mathrm{bc}$, we can see:

$$
\begin{gathered}
\mathrm{b}=\frac{\sum_{\mathrm{i}=1}^{\mathrm{n}}\left(\mathrm{c}_{\mathrm{i}}-\overline{\mathrm{c}}\right)\left(\mathrm{I}_{\mathrm{i}}-\overline{\mathrm{I}}\right)}{\sum_{\mathrm{i}=1}^{\mathrm{n}}\left(\mathrm{c}_{\mathrm{i}}-\overline{\mathrm{c}}\right)^{2}}=8621.43 \\
\mathrm{a}=\overline{\mathrm{I}}-\mathrm{b} \overline{\mathrm{c}}=3161.366
\end{gathered}
$$

the calibration equation $\mathrm{I}=8621.43 \mathrm{c}+3161.366$

So, uncertainty of element concentration in solution from Calibration curve linear fitting $u(c)_{1}$ is

$$
\mathrm{u}(\mathrm{c})_{1}=\frac{\mathrm{s}_{\mathrm{R}}}{\mathrm{b}} \sqrt{\frac{1}{\mathrm{~m}}+\frac{1}{\mathrm{n}}+\frac{\left(\mathrm{c}_{0}-\overline{\mathrm{c}}\right)^{2}}{\sum_{\mathrm{i}=1}^{\mathrm{n}}\left(\mathrm{c}_{\mathrm{i}}-\overline{\mathrm{c}}\right)^{2}}}
$$

$\mathrm{S}_{\mathrm{R}}$ means standard deviation of calibrated solution:

$$
\mathrm{S}_{\mathrm{R}}=\sqrt{\frac{\left.\sum_{\mathrm{i}=1}^{\mathrm{n}}\left(\mathrm{I}_{\mathrm{i}}-=1 \mathrm{c}_{\mathrm{i}}+\mathrm{a}\right)\right)^{2}}{\mathrm{n}-2}}=3042.577(\mathrm{~m}=9, \mathrm{n}=18)
$$

$\mathrm{u}(\mathrm{c})_{1}$ of element Cr was $0.145441 \mathrm{ug} / \mathrm{L}, \mathrm{u}_{\mathrm{rel}}(\mathrm{c})_{1}$ was 0.007 .

\subsubsection{Uncertainty of standard solution concentration: $u_{\text {rel }}(c)_{2}$}

The standard uncertainty: $\mathrm{u}_{\mathrm{rel}}(\mathrm{c})_{2}=2 \mathrm{mg} / \mathrm{L}$, so, the relative standard uncertainty of standard solution concentration: $u_{\text {rel }}(c)_{2}=0.002$.

\subsubsection{Uncertainty of removing the volume of standard solution: $u_{\text {rel }}(c)_{3}$}

According to GB/T 12805[5], The volume errors are $0, \pm 0.008, \pm 0.008, \pm 0.008, \pm 0.0080$, $\pm 0.050 \mathrm{ml}$, respectively, the corresponding standard uncertainty is $0,0.0033,0.0033,0.0033$, $0.0033,0.020 \mathrm{ml}$ based on the distribution of triangles, respectively:

$$
u_{\text {rel }}(c)_{3}=\sqrt{\left(0+0.033^{2}+0.0033^{2}+0.0033^{2}+0.0033^{2}+0.002^{2}\right) / 6}=0.0027
$$

\subsubsection{Relative standard uncertainty of element concentration in sample solution:}

$$
\mathrm{u}_{\mathrm{rel}}(\mathrm{c})=\sqrt{\mathrm{u}_{\mathrm{rel}}^{2}(\mathrm{c})_{1}+\mathrm{u}_{\mathrm{rel}}^{2}(\mathrm{c})_{2}+\mathrm{u}_{\mathrm{rel}}^{2}(\mathrm{c})_{3}}=0.008
$$

\subsection{Evalution of relative standard uncertainty of constant volume of sample solution: $u_{\text {rel }}(V)$}

According to GB/T 12806 and the distribution of triangles, The volume errors of $50 \mathrm{~mL}$ volumetric flask in class $A$ is $\pm 0.5 \mathrm{~mL}$. $\mathrm{u}(\mathrm{V})=0.02 \mathrm{ml}$ :

$$
u_{\text {rel }}(V)=0.020 / 50=0.0004
$$

4.3 Evalution of relative standard uncertainty resulted from weighing sample quality by balance: $\mathbf{u}_{\text {rel }}(\mathbf{m})$

We weigh the sample with a scale with a volume of $0.1 \mathrm{mg}$. The allowable difference is $\pm 0.1 \mathrm{mg}$,

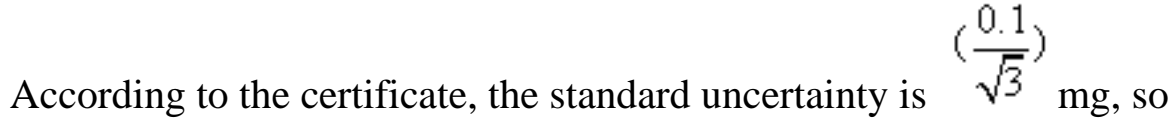




$$
u(m)=\sqrt{\left(\frac{0.1}{\sqrt{3}}\right)^{2} \times 2}=0.082(m g)
$$

and the relative standard uncertainty:

$$
u_{\text {rel }}(m)=0.082 / 500=0.00016
$$

\subsection{Evaluation of relative standard uncertainty introduced by repetitive experiments: $u_{\text {rel }}(\mathbf{r e p})$}

Weigh 3 samples, determined by the determination method, the results are shown in Table 1:

According to table 1, the standard uncertainty introduced by repetitive experiments is

$$
\begin{aligned}
\mathrm{u}(\text { rep })= & \mathrm{s}(\omega) / \sqrt{\mathrm{n}}=1437.6 \\
& \mathrm{u}_{\text {rel }}(\text { rep })=0.006 .
\end{aligned}
$$

Table1 Repeated results of the sample for three times

\begin{tabular}{ccc}
\hline $\begin{array}{c}\text { average } \\
\text { value }\end{array}$ & $\begin{array}{c}\text { Standard deviation of single } \\
\text { measurement }\end{array}$ & $\begin{array}{c}\text { The standard deviation of the average of the } \\
\text { measured values }\end{array}$ \\
\hline 276332 & 2490 & 1437.6 \\
\hline
\end{tabular}

\section{Evalution of synthetic standard uncertainty}

The components are not related to each other and the combined uncertainty is calculated according to the square root.

$$
\begin{array}{r}
u_{r e l}(w)=\sqrt{u_{r e l}^{2}(c)+u_{r e l}^{2}(V)+u_{r e l}^{2}(m)+u_{r e l}^{2}(r e p)}=0.01 \\
u(w)=2763^{u g} / \mathrm{kg}
\end{array}
$$

\section{Evalution of extended uncertainty}

$95 \%$ confidence interval, including factor $\mathrm{K}=2, U=2763 * 2=5527^{u g} / \mathrm{kg}$

\section{Expression of analysis results}

The content of Chromium by ICP-MS is:

$$
w=(276 \pm 6) \mathrm{mg} / \mathrm{kg}, k=2
$$

\section{Conclusions}

The content of element Chromium is $276 \pm 6 \mathrm{mg} / \mathrm{kg}, \mathrm{k}=2$, and we successfully established a uncertainty model for uncertainty evaluation based on regression equation, constant volume, weighing weight.

\section{References}

[1] Eriksen M, Maximenko N, Thiel M, et al. Plastic pollution in the South Pacific subtropical gyre. Marine Pollution Bulletin, 2013, 68(1-2):71-76.

[2] Azzarello M Y, Vleet E V. Marine birds and plastic pollution. Marine Ecology Progress, 1987, 37:295-303. 
[3] Eriksen M, Lebreton LC, Carson HS, et al. Plastic Pollution in the World's Oceans: More than 5 Trillion Plastic Pieces Weighing over 250,000 Tons Afloat at Sea. Plos One, 2014, 9(12):e111913.

[4] Information on https://www.cnas.org.cn/rkgf/sysrk/rkyyzz/2015/06/869093.shtml

[5] General Administration of Quality Supervision, Inspection and Quarantine of the People's Republic of China., GB/T 12805-2011 Laboratory glassware. 2012.. 